\title{
Solution of Modified Bergman's Minimal Blood Glucose Insulin Model Using Caputo- Fabrizio Fractional Derivative
}

\author{
RAVI DUBEY ${ }^{1}$, Dumitru Baleanu ${ }^{2}$, Manvendra Mishra ${ }^{3}$, and Pranay Goswami ${ }^{4}$ \\ ${ }^{1}$ Amity University Rajasthan \\ ${ }^{2}$ Cankaya University, \\ ${ }^{3}$ Amity University Jaipur \\ ${ }^{4}$ School of Liberal Studies, School of Liberal Studies, Ambedkar University Delhi-110006, \\ India
}

July 11, 2020

\begin{abstract}
In the current research work, we are going to discuss the Modified Bergman's Minimal blood glucose-insulin model.We are restructuring the old model by including one more component known as diet, which is also very essential for blood glucose model. Many years ago the minimal model was define which explain the relation between blood glucose and insulin level, and after that a lots of work and research has been done on it. We define the fractional model to show the strength of Caputo-Fabrizio fractional derivative and also examined the existence and uniqueness of the result along with its numerical and graphical representation.
\end{abstract}

\section{Hosted file}

blood.pdf available at https://authorea.com/users/330628/articles/468800-solution-ofmodified-bergman-s-minimal-blood-glucose-insulin-model-using-caputo-fabrizio-fractionalderivative 

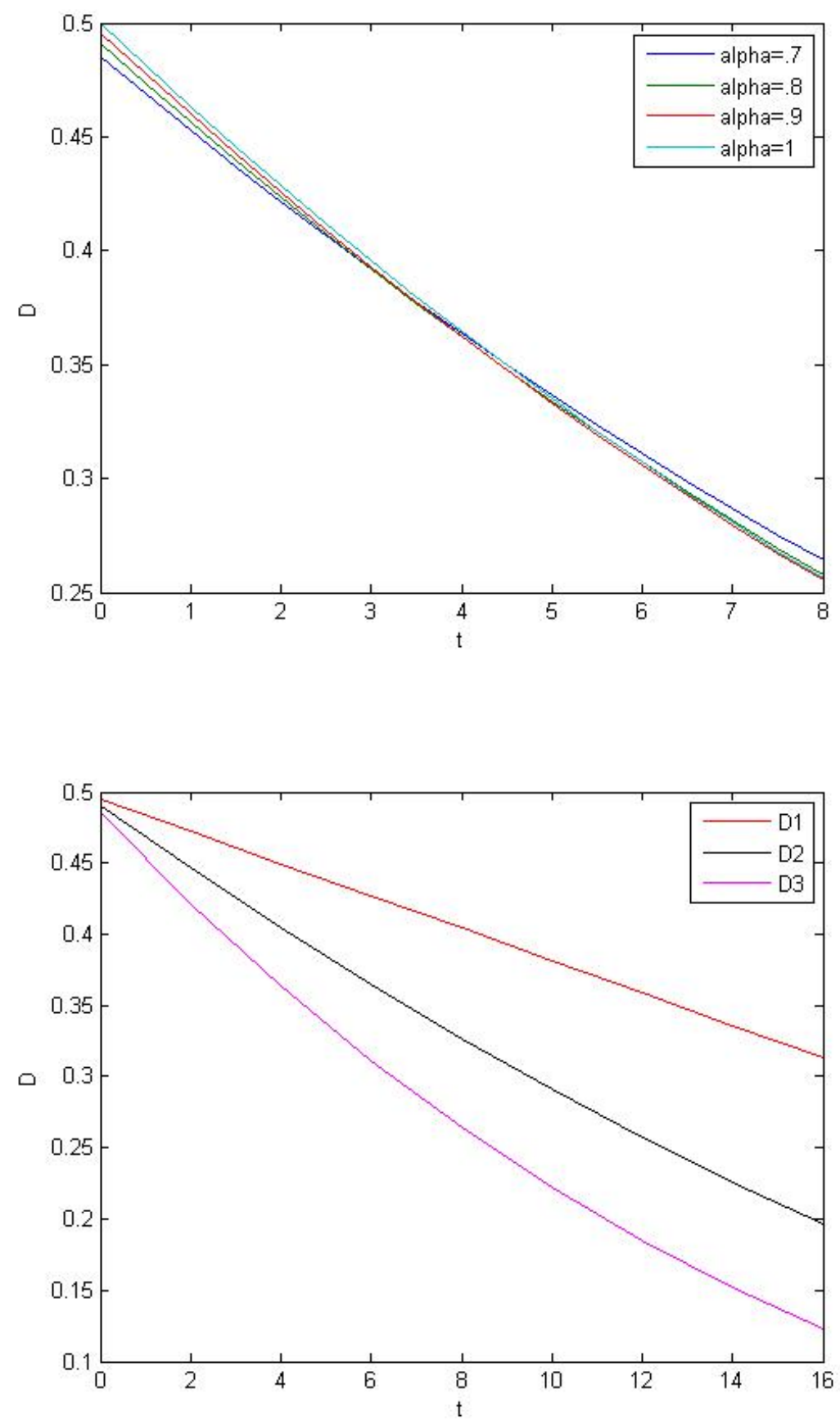

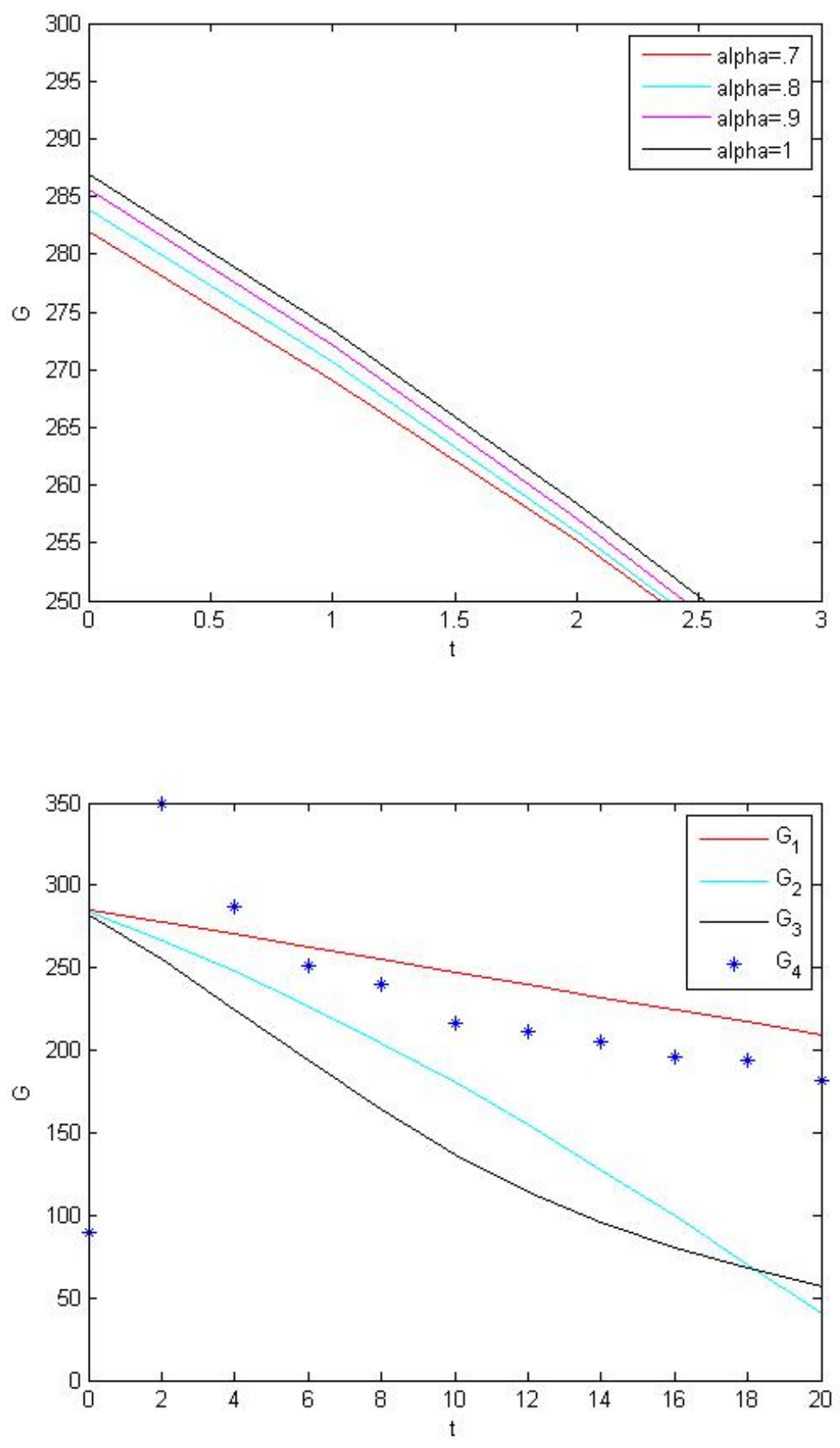

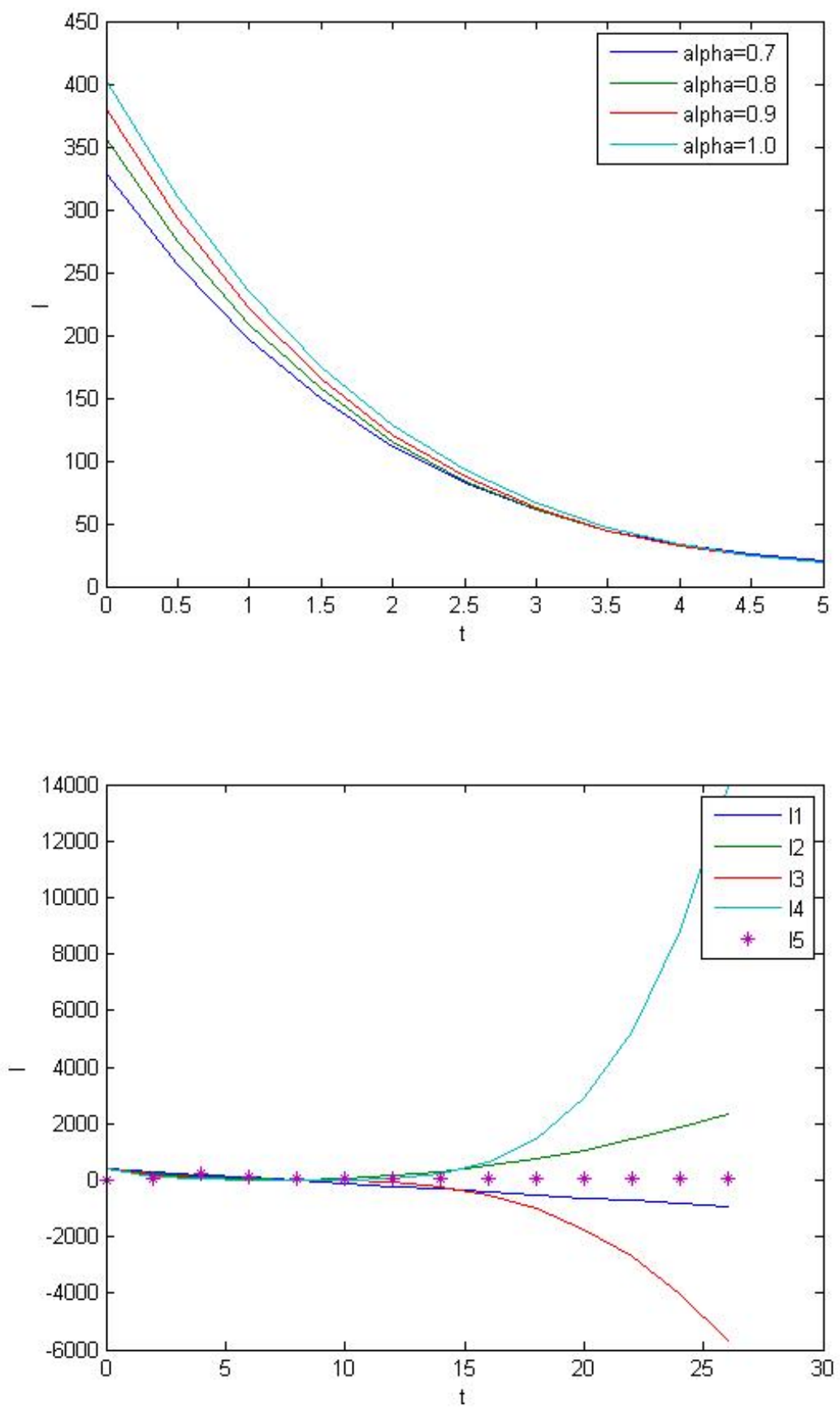

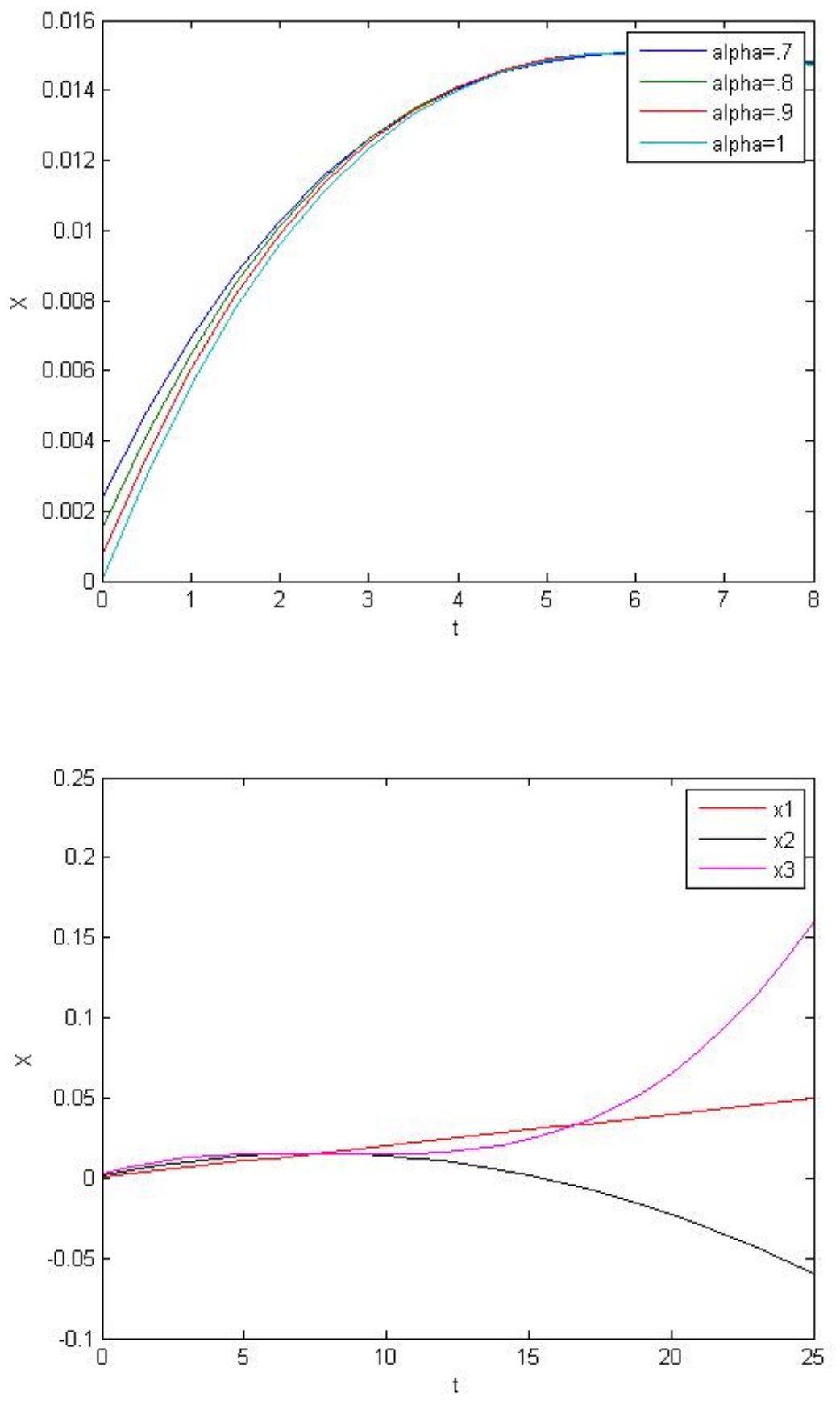